\title{
Structure Formation and Characterization in Supernova Explosions
}

\author{
Patrick A. Young ${ }^{1}$, Carola Ellinger ${ }^{2}$, Chris Fryer ${ }^{3}$, \\ and Gabe Rockefeller ${ }^{3}$ \\ ${ }^{1}$ School of Earth and Space Exploration, Arizona State University \\ P. O. Box 871404, Tempe Az, 85287, USA \\ email: patrick. young. 1Casu.edu \\ ${ }^{2}$ Department of Physics, University of Texas at Arlington \\ 502 Yates St. Science Hall Rm 108 Box 19059 Arlington, TX 76019, USA \\ email: ellinger@uta.edu \\ ${ }^{3}$ CCS Division, Los Alamos National Laboratory, \\ Los Alamos, NM 87545, USA \\ email: fryer@lanl.gov, gaber@lanl.gov
}

\begin{abstract}
Observations of supernova remnants show that large- and small scale structures form at various points in the explosion. We present a case study of structure formation in $3 \mathrm{D}$ in a $15 \mathrm{M}_{\odot}$ supernova for different parameters. We investigate the structure formation and morphology of the Rayleigh-Taylor unstable region. We also propose a method of characterizing the sizes of overdense clumps that can be compared directly with observations. The RT instabilities result in clumps that are overdense by 1-2 orders of magnitude with respect to the ambient gas, have size scales on the level of a few $\%$ of the remnant diameter, and are not diffused after the first $\sim 30$ yrs of the remnant evolution, in the absence of a surrounding medium.
\end{abstract}

Keywords. (ISM:) supernova remnants, diffusion, hydrodynamics, methods: numerical

\section{Introduction}

Morphological, kinematic, and compositional structures are ubiquitous in the observations of supernovae (SNe) and supernova remnants (SNRs). These structures span scales from unipolar asymmetries across the whole remnant to sub-AU (astronomical unit) sized high density knots in the reverse shock of Cassiopeia A. The dense knots in SNRs are of particular interest from a nucleosynthetic and astrobiological point of view as vehicles for the chemical enrichment of star and planet-forming material. In order to be able to make meaningful comparisons to remnants such as Cas A, where the evolution is dominated by the interaction of the ejecta with surrounding stellar winds and/or ISM, it is necessary to extend calculations to a much longer time after shock breakout. It is our aim to follow structures from their formation all the way to the young remnant phase.

\section{Simulations}

The simulations used a $15 \mathrm{M}_{\odot}$ progenitor of solar metallicity evolved to the onset of core collapse with the stellar evolution code TYCHO (Arnett, Meakin, \& Young 2009, Young et al. 2005, Arnett, Meakin \& Young 2009). To model collapse and explosion, we use a 1dimensional Lagrangian code (Herant et al. 1994, Fryer et al. 1999, Benz, Thielemann, \& Hills 1989). The explosion was followed until the revival of the shock, and then mapped into 3D when the shock had moved out of the Fe-core and propagated into the Si-S rich shell. We used the 3-D Lagrangian hydrodynamics code SNSPH (Fryer, Rockefeller, 
\& Warren 2006) to model the explosion. To accurately calculate the yields we turn to a post-process step performed with the Burn code (Young \& Fryer 2007). Radiative cooling is based on the tables from the CHIANTI atomic database for optically thin astrophysical plasmas, and assumes collisional ionization equilibrium. We did runs with imposed uni or bipolar explosion asymmetries implemented by modifying the velocities of particles in and inside of the shock according to the prescription in Hungerford et al. (2005). We repeated the $1 \mathrm{M}$ jet4 calculation once the shock reached the edge of the O-rich layer in a late asymmetry case $(1 \mathrm{M}$ jet4L) and another case for when the shock had propagated well into the $\mathrm{C}$ rich layer (1M jet4LL). We also computed a single-lobe scenario for each of the two jet asymmetries. In an effort to quantitatively describe the clump sizes, we calculated the power spectrum of the clump sizes in each simulation. The power spectrum versus wavelength will then show local maxima at the characteristic length scales of the clumps.

\section{Results}

In all simulations, the size scale for the shortest wavelengths indicated in all power spectrum plots is at $\sim 3 \%$ to $\sim 16 \%$ of the radius of the remnant. All spectra also show significant power at a length scale of 50 - $60 \%$ of the respective remnant size, which corresponds to the diameter of the shell of clumps created by the fluid instabilities. The broad peak at the largest wavelengths correlates with the size of the whole RT structure complex. The 50million particle run shows a peak between $0 R_{\odot}-200 R_{\odot}$ that is roughly Poisson-shaped and has a local maximum at $\sim 40 \mathrm{R}_{\odot}$. Another peak/double peak is centered at a length scale of $\sim 1 \times 10^{3} \mathrm{R}_{\odot}$, which correlates to the inner diameter of the RT clump structure. The maximum at $110 \mathrm{R}_{\odot}$ is associated with a broader peak between $50 \mathrm{R}_{\odot}$ and $150 \mathrm{R}_{\odot}$, followed by another peak at slightly below $200 \mathrm{R}_{\odot}$. Comparison with the slice through the original data suggests that the first peak between $50 \mathrm{R}_{\odot}-200$ $\mathrm{R}_{\odot}$ corresponds to the density clumps, whereas the peaks following it correspond mostly to the space in between those clumps. The peak at $40 \mathrm{R}_{\odot}$ in particular likely indicates size scales for the clumps themselves. The power spectra of the bipolar explosions show some differences to the symmetric runs. The most obvious is a peak, constituting the global maximum in each plot, at $\sim 3-9 \times 10^{5} \mathrm{R}_{\odot}$, which itself features 3 spikes. At short wavelengths, the bipolar runs show a series of distinct peaks out to $\sim 7.5 \times 10^{4} \mathrm{R}_{\odot}$. The three to four prominent peaks in the power spectra in that range mostly correspond to the added spacings between the ring of clumps and those inside of it, and the ring of clumps and the RT clumps outside of it. The power at wavelengths shorter than that range is likely still determined by the typical sizes of the clumps (RT and Ni bubble). In these simulations the Ni-bubble is clearly discernible, causing the different distributions of power at small length scales.

\section{References}

Arnett, David, Meakin, Casey, Young, \& Patrick, A. 2009, ApJ, 690, 1715

Benz, W., Thielemann, F.-K., \& Hills, J. G. 1989, ApJ, 342, 986

Fryer, Chris, Benz, Willy, Herant, Marc, Colgate, \& Stirling, A. 1999, ApJ, 516, 89

Fryer, C. L., Rockefeller, G., \& Warren, M. S. 2006, ApJ, 643, 292

Herant, M., Benz, W., Hix, W. R., Fryer, C. L., \& Colgate, S. A. 1994, ApJ, 435, 339

Hungerford, A. L., Fryer, C. L., \& Rockefeller, G. 2005, ApJ, 635, 487

Young, Patrick A., Arnett, \& David 2005, ApJ, 618, 908

Young, Patrick A., Meakin, Casey, Arnett, David, Fryer, \& Chris L. 2005, ApJL, 629, 101

Young, Patrick A., Fryer, \& Chris L. 2007, ApJ, 664, 1033 